\title{
High-volume image-guided injection in the chronic recalcitrant non-insertional patellar tendinopathy: a retrospective case series
}

\author{
Torsten Grønbech Nielsen * ${ }^{*}$, Lene Lindberg Miller, Bjarne Mygind-Klavsen and Martin Lind
}

\begin{abstract}
Purpose: To evaluate if High-volume Image-guided Injection (HVIGI)-treatment for chronic Patellar tendinopathy (PT) improve function and reduce pain at 16-months follow-up.

Methods: Patients with resistant PT who failed to improve after a three-month eccentric loading program were included in the study. Maximal tendon thickness was assessed with ultrasound. All patients were injected with 10 $\mathrm{mL}$ of $0.5 \%$ Marcaine, $0.5 \mathrm{~mL}$ Triamcinolonacetonid $(40 \mathrm{mg} / \mathrm{mL})$ and $40 \mathrm{~mL}$ of $0.9 \% \mathrm{NaCl}$ saline solution under realtime ultrasound-guidance and high pressure. All outcome measures were recorded at baseline and at 16 months. A standardised Heavy Slow Resistance rehabilitation protocol was prescribed after HVIGI-treatment. Clinical outcome was assessed with the Victorian Institute of Sports Assessment-Patella tendon questionnaire (VISA-P) and statistically analyses were performed.
\end{abstract}

Results: The study included 28 single treatment HVIGl procedures in PT in 23 patients (19 men, 4 women) with a mean age of 30.3 (range 19-52). The mean duration of symptoms before HVIGI was 33 months. The baseline VISA-P score of $43 \pm 17$ (range 15-76) improved to $76 \pm 16$ (range 42-95) after 16 months $(p<0.01$ ). Of the $28 \mathrm{HVIGl}$ procedures 12 patients (15 PT) were not satisfied after the initial HVIGI procedure. Of these, 5 patients (5 PT) had additional HVIGI, 2 patients (2 PT) had corticoid injection and 6 patients (8 PT) needed surgery. Of the remaining 11 patients (13 PT), 9 patients had more than a 13-point improvement in the VISA-P score after 16 months.

Conclusions: In this retrospective case-study, only 9 patients (32\%) did benefit of a single HVIGI treatment at 16-months and a 33-point significant improvement was seen on the VISA-P score.

Keywords: High-volume image-guided injection, HVIGl, Patellar tendinopathy, Patella tendon, Eccentric training, Heavy slow resistance training

\section{Background}

Jumper's knee or patellar tendinopathy (PT) is a common overuse injury among athletes. Overall prevalence of PT is seen in $10-14 \%$ of elite athletes. The prevalence is higher in sports with high demands on speed and power $[13,16,20]$. Lian et al. presented prevalence as high as $32-45 \%$ in elite volleyball and basketball players [20].

\footnotetext{
* Correspondence: torsne@rm.dk

Aarhus University Hospital, Orthopedic Department, Palle Juul-Jensens Boulevard 99, 8200 Aarhus N, Denmark
}

In nonelite athletes prevalence of PT is seen in 5.8$8.5 \%$ of athletes and again prevalence is higher in speed and power activities such as volleyball and basketball (range 11.3-14.4) [8, 24].

PT is a degenerative condition and could be characterized by a neovascularisation of the patella tendon $[15,18]$. The area of degeneration generally occurs distal of the inferior pole of the patella and in the proximal portion of the patella tendon [14].

In 2008, Chan et al. performed the first High-volume Image-guided Injection (HVIGI) study for chronic noninsertional achilles tendinopathy. HVIGI was used in a
Springer Open

(c) The Author(s). 2020 Open Access This article is licensed under a Creative Commons Attribution 4.0 International License, which permits use, sharing, adaptation, distribution and reproduction in any medium or format, as long as you give appropriate credit to the original author(s) and the source, provide a link to the Creative Commons licence, and indicate if changes were made. The images or other third party material in this article are included in the article's Creative Commons licence, unless indicated otherwise in a credit line to the material. If material is not included in the article's Creative Commons licence and your intended use is not permitted by statutory regulation or exceeds the permitted use, you will need to obtain permission directly from the copyright holder. To view a copy of this licence, visit http://creativecommons.org/licenses/by/4.0/. 
cohort of patients who failed a three-month guided eccentric training program (ET). The mechanism behind the effect of HVIGI-treatment is believed to be mechanical stretching, breaking or occluding the neovessels and the accompanying nerve ingrowth [9]. This is believed to reduce the pain of the tendinopathy.

Various treatment strategies for PT exist in the literature including eccentric training (ET), platelet rich plasma injections (PRP), shock wave, corticoid injection, load modification, electrolysis etc. These treatments have been performed with various results over the past decades $[2,4-7,10]$.

Two systematic reviews and meta-analyses from 2019 investigated non-surgical interventions ability to improve function and reduce pain in PT $[4,10]$ and concluded that PRP had the greatest improvement and pain reduction compared to the other non-surgical modalities in the long term.

No published HVIGI randomized controlled study (RCT) exists in the literature therefore no studies were included in the meta-analysis from 2019 [10]. A study from 2018 compares HVIGI with PRP and with HVIGI+ PRP. Of the three groups HVIGI+PRP is more effective in the short time than the other treatment [3].

Other treatment options on PT are presented in a consensus from ESSKA Basic Science Commitee (2018), which includes: ultrasound guided galvanic electrolysis technique (USGET), mesenchymal stem cells, gene therapy, biomaterials and surgical approach [1]. These treatments will not be presented further in this present study.

The aim of the current study was to evaluate if HVIGI-treatment for chronic PT improve function and reduce pain at 16-months follow-up.

It was hypothesised that HVIGI would result in clinically relevant improvements of symptoms and function for chronic Patellar tendinopathy after 16 months and that most of the patients would benefit from HVIGI treatment.

\section{Methods}

In this retrospective case series of 28 single HVIGI procedures followed prospectively were performed between May 2013 and October 2016. Patients were included if they had a diagnosis of tendinopathy (here defined by (1) pain localized to the inferior pole of patella and (2) neovascularisation and innervation in and around the tendon using ultrasound examination) and had failed a three-month Heavy Slow Resistance training (HSR) program [19]. Patients were excluded if they have had prior HVIGI treatment, patella surgery or were lost to follow up.

The Local Ethics Committee was contacted and determined that an approval for this study was not required (1-10-72-1-19). All data was managed with strict confidentiality and data has been anonymised prior to analysis. Three skilled orthopaedic surgeons diagnosed the patients on the basis of their medical history, physical examination and ultrasound evaluation.

\section{Intervention \\ Injection}

Patients were placed in the supine position with knee in full extension. The needle was inserted in the interface between the Hoffa's fat pad and the patella tendon. PT injection was performed with $10 \mathrm{~mL}$ of $0.5 \%$ Marcaine, $0.5 \mathrm{~mL}$ Triamcinolonacetonid $(40 \mathrm{mg} / \mathrm{mL})$ and $40 \mathrm{~mL}$ of $0.9 \% \mathrm{NaCl}$ saline solution under real-time ultrasound guidance aiming at the area of maximal neovascularisation.

\section{Rehabilitation}

In the first $72 \mathrm{~h}$ after the injection, patients were only allowed to participate in the normal activities of daily living. Running, jumping and heavy resistance training was prohibited. After $72 \mathrm{~h}$, patients were allowed to begin HSR as described by Kongsgaard [19]. A two-page leaflet with exercises and guidelines was given to the patient. Patients knew the rehabilitation due to a previously failed three-month rehabilitation program. If patients had the intention of returning to running and were pain-free, they were educated in a graded running program starting 1 month after HVIGI treatment.

\section{Clinical outcome measure}

The Victorian Institute of Sports Assessment-Patella tendon questionnaire (VISA-P) [23] was used as a primary outcome measure and was recorded at baseline and 16 months. This score ranged from 0 to 100 , where 0 was worst and 100 was asymptomatic. Primary outcome was the delta value between baseline and the 16months follow-up.

A successful outcome was defined as single HVIGI treatment, no subsequent other injection or conversion to surgery and a 13-points improvement [17] at the VISA-P at the 16-months follow-up.

\section{Ultrasound evaluation}

Maximal tendon thickness and neovascularisation were assessed with ultrasound and power Doppler in the supine position with full knee extension. The Patella tendon was scanned in both transverse and longitudinal planes. Ultrasound evaluation was performed at baseline and 16-months follow-up.

\section{Statistical analysis}

Descriptive statistics were calculated. Continuous data were presented as mean \pm standard deviation (SD). Categorical data were presented as quantity and proportions (\%). Normality was tested by qq-plots. All data were 
normally distributed, and the student's T-test was used to compare differences between time-points. Minimal clinical important difference (MCID) was set at 13 points [17]. $P$-values below 0.05 were considered significant. All data was analysed in MS Excel 2010 version 14.0.7237.5000 (32-bit) and STATA 16.1 software (StataCorp, College Station, TX, USA).

\section{Results}

Thirty-one single treatment HVIGI procedures were performed in the period 2013-2016. The study included $28 \mathrm{PT}$ in 23 patients (19 men, 4 women) (Fig. 1).

The mean age was 30.3 years (range 19-52). The mean duration of symptoms before HVIGI was 33 months (range 12-108).

Patient characteristics are listed in Table 1. Some patients had tried several other conservative treatments apart from HSR before HVIGI including corticosteroid injection or shock wave (Table 1).

The baseline VISA-P score of $43 \pm 17$ (range 15-76) improved significantly to $76 \pm 16$ (range $42-95$ ) at the 16 months follow-up $(p<0.01)$. VISA-P scores are listed in Table 2. Nine patients (32\%) having a single HVIGI treatment had above 13-point improvement.

The thickness of the Patella tendon measured at baseline was $7.1 \pm 1.3 \mathrm{~mm}$ compared to $6.1 \pm 2.0 \mathrm{~mm}$ at the 16 months follow-up $(p=0.10)$ (Table 2).

Of the 31 PT treated for a single treatment with HVIGI 3 patients (3 PT (10\%)) were lost to follow up.
Of the remaining $28 \mathrm{PT}$ (23 patients), 12 patients (15 PT (54\%)) were not satisfied after a single HVIGI procedure and required further treatment. Of these, 5 patients (5 PT) needed additional HVIGI, 2 patients (2 PT) needed corticoid injection and 6 patients (8 PT) needed surgery.

No complications in relation to HVIGI treatment were observed.

\section{Discussion}

The primary finding of the present study was that a successful outcome after a single HVIGI treatment in patients with PT who had previously failed exercise based treatment was seen in $32 \%$ of patients after 16 months. A significant improvement was demonstrated compared to the baseline VISA-P score. The improvement for successful treatments in the VISA-P score from baseline to the 16 months follow-up was 33 points.

Crisp, Morton, Maffulli and Abate found an improvement from baseline to $3-15$ months in their studies at $22,18,29$ and 10 points, respectively [3, 12, 21, 22].

The study from Crisp in 2008 was the first study presenting data from HVIGI procedures in PT [12]. Morton and Maffulli used the same cohort as Crisp (9 patients) in their respective studies from 2014 and 2016 [21, 22]. Differences between these 3 studies are the follow up time point varying from 3 to 15 months and VISA-P at the follow up time point. A tendency in these studies was that longer follow up period lead to better outcome.

\section{Single High-volume Image-guided Injection treatment ( $n=31$ Patella Tendons)}

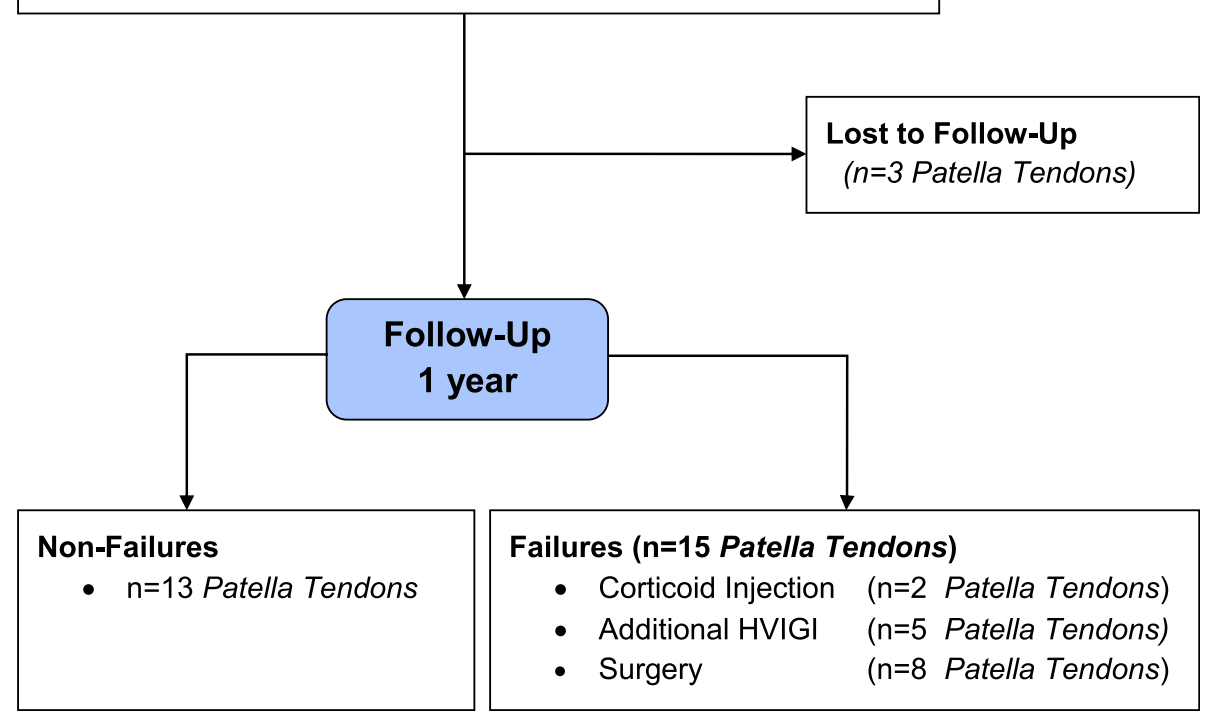

Fig. 1 Flow Chart 
Table 1 Patients demography

\begin{tabular}{ll}
\hline HVIGI procedures/patients, $\mathrm{n}$ & $28 / 23$ \\
Men/Women, \% & $83 / 17$ \\
Mean age in years (range) & $30.3(19-52)$ \\
Mean pain duration in months (range) & $33(12-108)$ \\
Intervention before HVIGI & \\
$\bullet \rightarrow$ Corticosteroid Injections, n (\%) & $17(61 \%)$ \\
$\bullet \rightarrow$ Shock Wave therapy, n (\%) & $1(4 \%)$ \\
\hline
\end{tabular}

Maffulli found a 29 points improvement at 15 months follow up, which was comparable with the present study, but patients were allowed to have more than one HVIGI treatment if first HVIGI treatment was insufficient. Twenty-five of the 44 patients (56\%) in their cohort had more than one HVIGI treatment and 3 patients were scheduled for surgery [21].

A difference between these studies and the present study could be the long duration of symptoms in the present patient cohort. Thirty-three months was the time between onset of Patella symptoms and HVIGI procedure compared to 3-21 months in the above-mentioned studies. Except for Abate's study, the 3 other studies used the HVIGI to recalcitrant PT with duration of symptoms of more than 18 months before HVIGI treatment.

In Abate's study, patients had not failed a three-month ET/HSR or tried other interventions. HVIGI was used as primary treatment and was performed twice with 14 days apart.

No difference between injection techniques and rehabilitation regimens was observed in the studies listed in Table 3.

Combining this issue of a high proportion of our cohort having previous corticosteroid treatment with the long duration of symptoms of mean 37 months, indicates that the HVIGI treatment in the present study served as a salvage procedure for PT when other treatments had failed. This could explain the low treatment response rate compared to previous studies in patients with less chronic conditions.

In the present study, a successful outcome was defined as one single HVIGI treatment, no subsequent conversion

Table 2 VISA-P and Patella tendon thickness

\begin{tabular}{llll}
\hline & Baseline & 16 Months & p-value \\
\hline Patella Tendons/Patients & $28 / 23$ & $13 / 11$ & \\
VISA-P completeness, n (\%) & $28(100)$ & $13(100)$ & \\
VISA-P, mean & $43 \pm 17$ & $76 \pm 16^{\mathrm{a}}$ & $<0.01$ \\
$>13$ points improvement, n (\%) & & $9(32)$ & \\
Patella tendon thickness, mm & $7.1 \pm 1.3$ & $6.1 \pm 2.0$ & 0.10 \\
\hline
\end{tabular}

Results are presented as mean-values with standard deviation ( \pm SD). ${ }^{\text {a }}$ significant improvement from baseline to surgery and a clinically relevant 13 point improvement at the VISA-P at the 16-months follow-up.

A successful outcome by Abate was a VISA-P improvement above 20 points from baseline to 6-months follow-up. Only 2 patients (11\%) had a successful outcome which is one third the success rate of the present study in which a $>20$ points improvement was seen in 9 patients (32\%).

Time from baseline to follow-up might be an important factor in the treatment of PT.

In the present study, a reduction of $1.0 \mathrm{~mm}$ in Patella tendon thickness after HVIGI treatment after 16 months was found. Other HVIGI studies have found reduced thickness of 1.0 to $1.2 \mathrm{~mm}$ at 3 and 15 months, respectively $[21,22]$.

It appears, based on the present study, that patients with long-lasting PT who have failed a three-month ET, corticosteroid injection or shockwave will have a $32 \%$ chance of a clinically relevant improvement in subjective outcome after a single HVIGI treatment after 16 months. These results indicate that patients with chronic PT and failed previous treatment should be counselled about the limited success rate of further treatment with HVIGI. This limited success rate could be explained by the pathomechanics in which patella tendon overload results in collagen degeneration, collagen disorganization and neovascularisation and innervation both in and around the tendon [11]. The patients have performed a 3 months specific training program before the HVIGI intervention with the intention to normalize the tendon tissue, as described by Kongsgaard [19]. As the patients did not benefit of the HSR training, HVIGI injection was performed with the purpose of peritendinous mechanical stretching, breaking the fibrosis and neovessels and the accompanying nerve ingrowth. HVIGI treatment does not address the intra tendinous pathologic changes and this could be a main factor for why only $32 \%$ of the patient had a clinical relevant improvement at 16 months follow-up.

The HVIGI treatment is found safe, due to no complications observed regarding this cohort.

Several limitations were acknowledged in this study. The most important were related to the absence of a control group. Also training compliance is unknown due to the lack of a training dairy. Vascularisation and neovascularisation were not measured at baseline and 16 months. Functional testing such as; gait analysis, strength assessment etc. could be beneficial in the analysis of the outcome after the HVIGI intervention. Another limitation could be $\mathrm{NaCl}$ saline solution in combination with Triamcinolonacetonid (cortisone) which could have an influence on the results, despite the dose of Triamcinolonacetonid was only $20 \mathrm{mg}$ per HVIGI procedure. 
Table 3 Studies presenting data on High-volume Image-guided Injection (HVIGl)

\begin{tabular}{llllllllll}
\hline & Pts $(\mathbf{n})$ & Age (y) & DoS (mt) & Post (mt) & US $\boldsymbol{\Delta}(\mathbf{m m})$ & VISA-P pre & VISA-P post & $\begin{array}{c}\text { VISA-P } \boldsymbol{\Delta} \\
\text { ET/HSR } \\
\text { failed }\end{array}$ \\
\hline Crisp [12] (2008) & 9 & 29 & 21 & 9 & $/$ & 46 & 68 & 22 & YES \\
Morton [22] (2014) & 20 & 31 & 20 & 3 & 1.0 & 46 & 64 & 18 & YES \\
Maffulli [21] (2016) & 44 & 35 & 18 & 15 & 1.2 & 46 & 75 & 29 & YES \\
Abate [3] (2018) & 30 & 37 & 3 & 6 & $/$ & 53 & 63 & 10 & NO \\
\hline
\end{tabular}

VISA-P is presented as mean-values with standard deviation $( \pm \mathrm{SD})$. Pts $=$ patients, $\mathrm{mt}=$ months, DoS $=$ Duration of symptoms, Post $=$ Latest follow-up, US $=$ tendon thickness between baseline and Latest follow-up, $\Delta=$ improvement from pre to post, ET/HSR failed = Eccentric Traning/HSR failed before HVIGI, /=unknown

\section{Conclusion}

In this study $32 \%$ of the patients with chronic PT who had failed Heavy slow resistance training treatment did benefit of a single HVIGI treatment. Among the nonfailures a significant 33-point improvement in VISA-P score was seen at 16-months follow-up.

\section{Abbreviations}

PT: Patellar Tendinopathy; HVIGI: High-volume Image-guided Injection; ET: Eccentric training; PRP: Platelet Rich Plasma; RCT: Randomized controlled study; HSR: Heavy Slow Resistance Training; VISA-P: Victorian Institute of Sports Assessment-Patella tendon questionnaire; MCID: Minimal clinical important difference

\section{Acknowledgements}

Not applicable.

\section{Authors' contributions}

BK diagnosed the patients and performed the HVIGI. LM and TN instructed the patients and completed the 16 months follow up. Data analysis was done by TN. Analysis and interpretation of the results was done by all the authors. ML was a major contributor in writing the manuscript. All authors read and approved the final manuscript.

\section{Funding}

No funding.

\section{Availability of data and materials}

The datasets used and/or analysed during the current study are available from the corresponding author on reasonable request.

\section{Ethics approval and consent to participate}

The Local Ethics Committee was contacted and determined that an approval for this study was not required (1-10-72-1-19).

\section{Consent for publication}

Not applicable.

\section{Competing interests}

The authors declare that they have no competing interests.

Received: 11 August 2020 Accepted: 5 October 2020

Published online: 10 October 2020

\section{References}

1. Abat F, Alfredson $\mathrm{H}$, Cucchiarini M, Madry H, Marmotti A, Mouton C et al (2018) Current trends in tendinopathy: consensus of the ESSKA basic science committee. Part II: treatment options. J Exp Orthop 5:38

2. Abat F, Sanchez-Sanchez JL, Martin-Nogueras AM, Calvo-Arenillas II, Yajeya J, Mendez-Sanchez R et al (2016) Randomized controlled trial comparing the effectiveness of the ultrasound-guided galvanic electrolysis technique (USGET) versus conventional electro-physiotherapeutic treatment on patellar tendinopathy. J Exp Orthop 3:34

3. Abate M, Di Carlo L, Verna S, Di Gregorio P, Schiavone C, Salini V (2018) Synergistic activity of platelet rich plasma and high volume image guided injection for patellar tendinopathy. Knee Surg Sports Traumatol Arthrosc 26: 3645-3651

4. Andriolo L, Altamura SA, Reale D, Candrian C, Zaffagnini S, Filardo G (2019) Nonsurgical treatments of patellar Tendinopathy: multiple injections of platelet-rich plasma are a suitable option: a systematic review and metaanalysis. Am J Sports Med 47:1001-1018

5. Backman LJ, Danielson P (2011) Low range of ankle dorsiflexion predisposes for patellar tendinopathy in junior elite basketball players: a 1-year prospective study. Am J Sports Med 39:2626-2633

6. Basas A, Cook J, Gomez MA, Rafael MA, Ramirez C, Medeiros B et al (2018) Effects of a strength protocol combined with electrical stimulation on patellar tendinopathy: 42 months retrospective follow-up on 6 high-level jumping athletes. Phys Ther Sport 34:105-112

7. Biernat R, Trzaskoma Z, Trzaskoma L, Czaprowski D (2014) Rehabilitation protocol for patellar tendinopathy applied among 16- to 19-year old volleyball players. J Strength Cond Res 28:43-52

8. Cassel M, Baur H, Hirschmuller A, Carlsohn A, Frohlich K, Mayer F (2015) Prevalence of Achilles and patellar tendinopathy and their association to intratendinous changes in adolescent athletes. Scand J Med Sci Sports 25: e310-e318

9. Chan O, O'Dowd D, Padhiar N, Morrissey D, King J, Jalan R et al (2008) High volume image guided injections in chronic Achilles tendinopathy. Disabil Rehabil 30:1697-1708

10. Chen PC, Wu KT, Chou WY, Huang YC, Wang LY, Yang TH et al (2019) Comparative effectiveness of different nonsurgical treatments for patellar Tendinopathy: a systematic review and network meta-analysis. Arthroscopy 35(3117-3131):e3112

11. Cook JL, Purdam CR (2009) Is tendon pathology a continuum? A pathology model to explain the clinical presentation of load-induced tendinopathy. Br J Sports Med 43:409-416

12. Crisp T, Khan F, Padhiar N, Morrissey D, King J, Jalan R et al (2008) High volume ultrasound guided injections at the interface between the patellar tendon and Hoffa's body are effective in chronic patellar tendinopathy: a pilot study. Disabil Rehabil 30:1625-1634

13. Durcan L, Coole A, McCarthy E, Johnston C, Webb MJ, O'Shea FD et al (2014) The prevalence of patellar tendinopathy in elite academy rugby: a clinical and imaging study. J Sci Med Sport 17:173-176

14. Figueroa D, Figueroa F, Calvo R (2016) Patellar Tendinopathy: diagnosis and treatment. J Am Acad Orthop Surg 24:e184-e192

15. Gisslen $\mathrm{K}$, Alfredson $\mathrm{H}$ (2005) Neovascularisation and pain in jumper's knee: a prospective clinical and sonographic study in elite junior volleyball players. Br J Sports Med 39:423-428 discussion 423-428

16. Gisslen K, Gyulai C, Soderman K, Alfredson H (2005) High prevalence of jumper's knee and sonographic changes in Swedish elite junior volleyball players compared to matched controls. Br J Sports Med 39:298-301

17. Hernandez-Sanchez S, Hidalgo MD, Gomez A (2014) Responsiveness of the VISA-P scale for patellar tendinopathy in athletes. Br J Sports Med 48:453457

18. Hoksrud A, Ohberg L, Alfredson H, Bahr R (2008) Color Doppler ultrasound findings in patellar tendinopathy (jumper's knee). Am J Sports Med 36: 1813-1820

19. Kongsgaard M, Kovanen V, Aagaard P, Doessing S, Hansen P, Laursen AH et al (2009) Corticosteroid injections, eccentric decline squat training and heavy slow resistance training in patellar tendinopathy. Scand J Med Sci Sports 19:790-802

20. Lian OB, Engebretsen L, Bahr R (2005) Prevalence of jumper's knee among elite athletes from different sports: a cross-sectional study. Am J Sports Med 33:561-567 
21. Maffulli N, Del Buono A, Oliva F, Testa V, Capasso G, Maffulli G (2016) Highvolume image-guided injection for recalcitrant patellar Tendinopathy in athletes. Clin J Sport Med 26:12-16

22. Morton S, Chan O, King J, Perry D, Crisp T, Maffulli N et al (2014) High volume image-guided injections for patellar tendinopathy: a combined retrospective and prospective case series. Muscles Ligaments Tendons J 4 : 214-219

23. Visentini PJ, Khan KM, Cook JL, Kiss ZS, Harcourt PR, Wark JD (1998) The VISA score: an index of severity of symptoms in patients with jumper's knee (patellar tendinosis). Victorian Institute of Sport Tendon Study Group. J Sci Med sport 1:22-28

24. Zwerver J, Bredeweg SW, van den Akker-Scheek I (2011) Prevalence of Jumper's knee among nonelite athletes from different sports: a crosssectional survey. Am J Sports Med 39:1984-1988

\section{Publisher's Note}

Springer Nature remains neutral with regard to jurisdictional claims in published maps and institutional affiliations.

\section{Submit your manuscript to a SpringerOpen ${ }^{\circ}$ journal and benefit from:}

- Convenient online submission

- Rigorous peer review

- Open access: articles freely available online

- High visibility within the field

- Retaining the copyright to your article

Submit your next manuscript at $\boldsymbol{\triangleright}$ springeropen.com 